This is a postprint version of the following article: Portero-Tresserra, M. ; Del Olmo, N. ; MartíNicolovius, M. ; Guillazo-Blanch, G. ; Vale-Martínez, A. (2014) D-cycloserine prevents relational memory deficits and suppression of long-term potentiation induced by scopolamine in the hippocampus. European Neuropsychopharmacology. Vol. 24, issue 11, p. 1798-1807. ISSN: 0924977X DOI: $\underline{10.1016 / j . e u r o n e u r o .2014 .10 .002}$

\title{
D-cycloserine prevents relational memory deficits and suppression of long-term potentiation induced by scopolamine in the hippocampus
}

\author{
Marta Portero-Tresserra ${ }^{1}$ \\ Nuria Del Olmo ${ }^{2}$ \\ Margarita Martí-Nicolovius ${ }^{1}$ \\ Gemma Guillazo-Blanch ${ }^{1}$ \\ Anna Vale-Martínez
}

Affiliation of all authors:

(1) Departament de Psicobiologia i Metodologia de les Ciències de la Salut, Institut de Neurociències, Universitat Autònoma de Barcelona, Barcelona, Spain.

(2) Departamento de Ciencias Farmacéuticas y de la Salud, Facultad de Farmacia, Universidad Ceu-San Pablo, Madrid, Spain.

Running head: Intra-hippocampal DCS reverses SCOP-induced deficits in memory and LTP

Total word/count: 6424

Address for correspondence:

Dr. Anna Vale-Martínez

Departament de Psicobiologia i Metodologia de les Ciències de la Salut,

Institut de Neurociències, Universitat Autònoma de Barcelona,

Barcelona (Spain).

Email: $\underline{\text { Anna.Vale@uab.cat }}$

Telephone: 34935813260 / Fax: 34935812001 


\section{ABSTRACT}

Previous research has demonstrated that systemic D-cycloserine (DCS), a partial agonist of the Nmethyl-D-aspartate receptor (NMDAR), enhances memory processes in different learning paradigms and attenuates mnemonic deficits produced by diverse pharmacological manipulations. In the present study two experiments were conducted in rats to investigate whether DCS administered in the hippocampus may rescue relational memory deficits and improve deficient synaptic plasticity, both induced by an intracerebral injection of the muscarinic receptor antagonist scopolamine (SCOP). In experiment 1, we assessed whether DCS would prevent SCOP-induced amnesia in an olfactory learning paradigm requiring the integrity of the cholinergic system, the social transmission of food preference (STFP). The results showed that DCS (10 $\mu \mathrm{g} / \mathrm{site})$ injected into the ventral hippocampus (vHPC) before STFP acquisition compensated the 24-h retention deficit elicited by post training intravHPC SCOP $(40 \mu \mathrm{g} / \mathrm{site})$, although it did not affect memory expression in non-SCOP treated rats. In experiment 2, we evaluated whether the perfusion of DCS in hippocampal slices may potentiate synaptic plasticity in CA1 synapses and thus recover SCOP-induced deficits in long-term potentiation (LTP). We found that DCS $(50 \mu \mathrm{M}$ and $100 \mu \mathrm{M})$ was able to rescue SCOP $(100 \mu \mathrm{M})$-induced LTP maintenance impairment, in agreement with the behavioral findings. Additionally, DCS alone $(50 \mu \mathrm{M}$ and $100 \mu \mathrm{M}$ ) enhanced field excitatory postsynaptic potentials prior to high frequency stimulation, although it did not significantly potentiate LTP. Our results suggest that positive modulation of the NMDAR, by activation of the glycine-binding site, may compensate relational memory impairments due to hippocampal muscarinic neurotransmission dysfunction possibly through enhancements in LTP maintenance.

Keywords: acetylcholine; glutamate; NMDA receptors; muscarinic receptors; relational memory; synaptic plasticity. 


\section{INTRODUCTION}

One of the most relevant targets of neuroenhancement strategies is the N-methyl-D-aspartate receptor (NMDAR), which is the predominant site for inducing learning-related synaptic plasticity in the hippocampus (Lee and Silva, 2009). D-cycloserine (DCS), a partial agonist of the NMDAR that binds to the glycine-site and enhances receptor activation in the presence of glutamate (Norberg et al. 2008), has recently been recognized for its potential in facilitating different cognitive processes.

Studies in rodents indicate that DCS, administered systemically or intracerebrally, improves numerous learning and memory processes in several paradigms (for reviews see Portero-Tresserra et al., 2013b; Villarejo-Rodríguez et al., 2010). Furthermore, DCS attenuates mnemonic deficits in aged (Aura and Riekkinen, 2000) and stressed rats (Yamamoto et al., 2008), and also in brain damaged rodents (Villarejo-Rodríguez et al., 2013; Yaka et al., 2007). Evidence from studies in humans suggests that DCS promotes both the consolidation and extinction of conditioned fear (Kalisch et al., 2009) and may also enhance declarative learning (Onur et al., 2010). Furthermore, it has also been shown that DCS improved cognition in Alzheimer's disease patients (Tsai et al., 1999).

Moreover, it has been shown that DCS antagonized memory decreases produced by the muscarinic acetylcholine (ACh) receptor antagonist scopolamine (SCOP), which may be considered a pharmacological model for cholinergic cognitive impairment (Klinkenberg and Blokland, 2010). Thus, the acute systemic pre-learning DCS infusion attenuated SCOP-induced deficits in acquisition of spatial tasks (Pitkänen et al., 1995) and brightness discrimination (Andersen et al., 2002). As for intracerebral administration, early studies showed that the hippocampus may be a relevant area since DCS microinfusions into its dorsal portion ameliorated SCOP-induced spatial working memory impairment ( Kishi et al., 1998; Ohno and Watanabe, 1996). Nevertheless, the physiological mechanisms by which such effects may occur have been poorly investigated. Several data suggest that DCS may improve SCOP-disrupted memory by acting on synaptic plasticity, and particularly longterm potentiation (LTP). On the one hand, SCOP administration impaired LTP in the CA1 hippocampal field (Calabresi et al., 1999; Sánchez et al., 2009) and altered hippocampal glutamate 
receptor levels (Falsafi et al., 2012). On the other hand, DCS may enhance memory by facilitating NMDAR-dependent synaptic potentials and CA1 synaptic plasticity (Rouaud and Billard, 2003; Billard and Rouaud, 2007). Accordingly, DCS reinstated LTP in CA1 and improved memory and neurological impairments in both brain-damaged (Yaka et al., 2007) and neural cell adhesion molecule-deficient mice (Kochlamazashvili et al., 2012).

In this context, the aim of the present study was to assess whether intra-hippocampal DCS may prevent deficits elicited by muscarinic blockade, both at a behavioral and physiological level. Thus, in the first experiment, we sought to confirm whether an acute DCS pre-learning treatment, as has been reported with intra-prelimbic cortex (PLC) administration (Portero-Treserra et al., 2013b), reverses SCOP-induced deficits in social transmission of food preference (STFP), which is a paradigm of social learning involving an ethologically meaningful test of olfactory memory that is considered a relational memory test with no explicit spatial components (Eichenbaum, 2000). STFP requires the integrity of the cholinergic system (Vale-Martinez et al., 2002) specifically in the ventral hippocampus (vHPC) (Carballo-Marquez et al., 2007), where a substantial increase in ACh release has been demonstrated after learning (Gold et al., 2011). The relevance of the vHPC has also been demonstrated in studies showing enhanced c-fos activity (Ross and Eichenbaum, 2006) and CREB phosphorylation (Countryman et al., 2005) in STFP learning. In the second experiment, we explored whether DCS may rescue putative deficits induced by SCOP in the induction/maintenance of LTP. To that end, electrophysiological recordings in CA1 synapses were carried out under the influence of DCS and SCOP perfusions (alone and in combination) in hippocampal slices both before and after a high frequency stimulation (HFS) protocol.

\section{EXPERIMENTAL PROCEDURES}

\section{Experiment 1}

\section{Subjects}

Fifty male Wistar rats, obtained from our laboratory breeding stock (Prolabor, Charles River Laboratories, Arbresle, France), with a mean age of 94.53 days $(\mathrm{SD}=6.231)$ and a mean weight of $402.795 \mathrm{~g}(\mathrm{SD}=33.86)$ at the beginning of the experiment, were used as observer subjects. An 
additional set of 36 male Wistar rats (mean age $=57.25 \mathrm{~d}, \mathrm{SD}=4.89$; mean weight $225.17 \mathrm{~g}, \mathrm{SD}=47.55$ ) served as demonstrator subjects. Juvenile demonstrator rats were used in order to avoid fighting and favor social interaction (Vale-Martinez et al., 2002). Procedures for housing, food restriction and habituation are explained in detail elsewhere (Portero-Tresserra et al., 2013a). All procedures were carried out in compliance with the European Community Council Directive for care and use of laboratory animals (86/609/ECC) and with the Generalitat de Catalunya authorization (DOGC 2450 7/8/1997, DARP protocol number 5959).

\section{Surgery}

Observer rats were anesthetized with isoflurane and underwent stereotaxic implantation of bilateral chronic guide cannulae in the vHPC: AP, $-5.0 \mathrm{~mm}$; ML, $\pm 5.0 \mathrm{~mm}$; and DV, $-6.8 \mathrm{~mm}$ (Paxinos and Watson, 1997), following procedures explained in detail elsewhere (Carballo-Marquez et al., 2009). After surgery, rats were returned to their home cages for 10 days (four for recovery, four for food restriction and two for rehabituation to ground food) before behavioral training.

\section{Microinfusion procedure}

The rats received 10 $\mu \mathrm{g} / \mathrm{hemisphere} \mathrm{of} \mathrm{DCS} \mathrm{(Sigma-Aldrich,} \mathrm{Madrid,} \mathrm{Spain)} \mathrm{or} \mathrm{phosphate-}$ buffered saline (PBS; 0.1M, pH 7.4) infusions $20 \mathrm{~min}$ before STFP acquisition and 40 $\mu \mathrm{g} /$ hemisphere SCOP (Scopolamine Hydrobromide USP, Sigma-Aldrich, Madrid, Spain) or PBS immediately after the social learning session. The solutions were infused bilaterally in a volume of $0.5 \mu \mathrm{l} /$ hemisphere for $2 \mathrm{~min}$ (plus $1 \mathrm{~min}$ to allow for diffusion) following procedures described previously (PorteroTresserra, et al., 2013a). The dose of DCS selected has been established previously to facilitate olfactory learning tasks when injected into the PLC or the basolateral amygdala (Portero-Tresserra et al., 2013a,b; Villarejo-Rodríguez et al., 2010), and other learning tasks, such as extinction of fear conditioning and appetitive learning, when injected into the basolateral amygdala or the infralimbic cortex (Peters \& de Vries 2013; Walker et al. 2002). The SCOP dose was chosen on the basis of a pilot study showing that intra vHPC injections of $40 \mu \mathrm{g}$ of SCOP disrupted STFP memory consolidation without inducing motor alterations (unpublished data).

\section{Behavioral procedure}

\section{Habituation to food jars}


The apparatus for STFP habituation, acquisition and testing is described in more detail in Portero-Tresserra et al. (2013b). After seven days of food restriction and prior to surgery, observers and demonstrators were habituated for three days to powdered chow (Scientific Animal Food and Engineering, Augy, France). A similar procedure was repeated $7 \mathrm{~d}$ after surgery for the observers (two 45-min rehabituation sessions). Subsequently, animals were again food-restricted for two days before the training-testing sessions began.

\section{STFP acquisition and test}

The STFP training followed procedures explained elsewhere (Portero-Tresserra et al., 2013a). Essentially, the task began when a demonstrator was allowed to eat food flavored with $2.2 \%$ cocoa (Oxfam Fairtrade, Gent, Belgium) or 1\% cinnamon (Carmencita, Alicante, Spain) for 30 min. Immediately after, observers and demonstrators interacted for $30 \mathrm{~min}$. All observers were tested $24 \mathrm{~h}$ after acquisition (45-min test) on the basis of previous studies showing amnesic effects after such a time period in SCOP-treated rats (Carballo-Marquez et al., 2009). A preference score (percentage of trained food) was calculated as follows: $100 \mathrm{x}$ (weight of trained food eaten/weight of all food eaten).

The number of times each observer sniffed the muzzle, body or anogenital region of the demonstrator, fighting and grooming (social interaction), and the number of forepaws (jar climbs during STFP test) were recorded (JVC, Everio Model GZ-X900). To rule out olfactory alterations due to the DCS and SCOP infusions, an additional olfactory perception test (described in Portero-Tresserra et al., 2013a) was conducted.

\section{Histology}

Perfusions and brains' post fixation were performed as explained elsewhere (CarballoMarquez et al., 2009). Coronal 40- $\mu \mathrm{m}$ sections were cut on a cryostat (Shandom Cryotome FSE, Thermo Electron Corporation, Massachusetts, USA), mounted and processed for acetylcholinesterase histochemistry and examined essentially as described previously (Portero-Tresserra et al., 2013a).

\section{Data analysis}

The main analysis of the variable percentage of trained food was performed by means of ANOVA (PASW v20) with the group factor as the independent variable (VEH, DCS, SCOP and 
DCS+SCOP). Specific between-group comparisons were performed and the Bonferroni correction was used. In addition, a one-sample t test against a constant (50) was used for each group to determine whether the percentage of trained food eaten was different from the chance level (50\%).

To evaluate whether all the animals had similar opportunities of learning (similar social interaction levels), we carried out an ANOVA considering group as the independent variable, and the dependent variables were sniffs of demonstrator's muzzle, body and anogenital region, fighting and grooming. Pearson correlation tests were used in order to examine the relationship between such variables and the percentage of trained food selected. Additional ANOVAs were also used to analyze total food eaten and jar climbs, which evaluated motivation to eat and explore, respectively. Mixed analyses of variance were carried out to analyze neophobia, with regular food (mean $\mathrm{g}$ of food eaten during the last rehabituation session prior to training) and new food (mean $\mathrm{g}$ of total food eaten, trained + untrained, during the test) as dependent variables.

Regarding the olfactory test, an ANOVA was applied considering group (VEH, DCS, SCOP and DCS+SCOP) as the independent variable, and latency in finding the buried cookie as the dependent variable.

\section{Experiment 2}

\section{Subjects}

Electrophysiological assays were carried out in 10-week old male Wistar rats (Prolabor, Charles River Laboratories, Arbresle, France) in accordance with the European Communities Council Directive (86/609/EEC) for the care and use of laboratory animals.

\section{Electrophysiological recordings in hippocampal slices}

Procedures of the electrophysiological experiments are precisely described in Miguéns et al. (2011). Drugs (DCS and SCOP) were prepared as stock solutions in PBS, stored frozen in the dark, and diluted to final concentration immediately before use. After obtaining stable synaptic responses

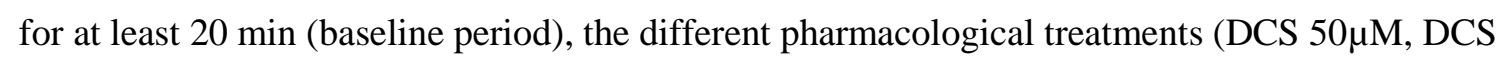
$100 \mu \mathrm{M}$, SCOP $100 \mu$, DCS $50 \mu \mathrm{M}+\operatorname{SCOP} 100 \mu \mathrm{M}$ and DCS $100 \mu \mathrm{M}+\operatorname{SCOP} 100 \mu \mathrm{M})$ were perfused for $40 \mathrm{~min}$. After $20 \mathrm{~min}$ of drug perfusion, Schaffer collateral fibers were tetanized with three $100-\mathrm{Hz}$ 
pulses of $100 \mu$ s duration every $20 \mathrm{~s}$ (HFS) to induce saturated-LTP. Following LTP induction, recording was carried out for $60 \mathrm{~min}, 20 \mathrm{~min}$ with drug and $40 \mathrm{~min}$ with KRB solution alone.

\section{Data analysis}

Data are expressed as the mean \pm SEM. The mean values of the initial slope of the fEPSP correspond to the averages of $1 \mathrm{~min}$ intervals. In all experiments potentiation was assessed by analyzing the mean slope of the fEPSP measured at different time points after HFS application. A value of $p<0.05$ was considered statistically significant.

To evaluate whether the administration of the different drugs produced changes in the basal synaptic transmission, one-way ANOVAs (PASW v20) were performed in order to compare the mean of the fEPSP slope for 20 min before HFS of each experimental group (DCS 50 $\mu \mathrm{M}$, DCS $100 \mu \mathrm{M}$,

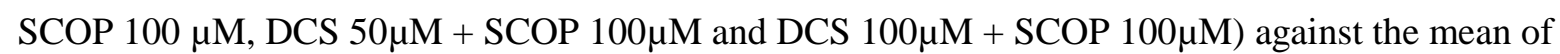
the fEPSP slope during the 20-min baseline of the VEH group. To evaluate the effects of the drugs on LTP, a mixed ANOVA was carried out, in which the between-subject factor was group (VEH, DCS $50 \mu \mathrm{M}$, DCS $100 \mu \mathrm{M}, \mathrm{SCOP} 100 \mu \mathrm{M}, \mathrm{DCS} 50 \mu \mathrm{M}+\mathrm{SCOP} 100 \mu \mathrm{M}$ and DCS $100 \mu \mathrm{M}+\operatorname{SCOP} 100 \mu \mathrm{M})$ and the within-subject factor was time (the last 10 minutes of the recording). Finally, the percentage of potentiation in the final recording (percentage of potentiation during the last $10 \mathrm{~min}$ of recording in relation to $10 \mathrm{~min}$ of the basal line prior to HFS) was considered as the dependent variable and the group factor as the independent variable (which included 6 categories). Contrast analyses were performed by means of Fisher's LSD. Differences were considered significant when $\mathrm{p}<0.05$.

\section{RESULTS}

\section{Experiment 1}

\section{Histology}

For the final sample we only considered rats with their cannula tips bilaterally located in the vHPC within the area delimited by CA3 and CA1, and the cannulae were located from -4.52 to -5.20 mm posterior to bregma (Figure 1). Eight rats were excluded from behavioral data analyses since their cannulae were incorrectly implanted $(n=5)$ or due to technical problems during the infusion $(n=3)$. 
Thus, the final sample was made up of 42 subjects distributed into the following groups: VEH ( $\mathrm{n}=10)$, $\operatorname{DCS}(n=10), \operatorname{SCOP}(n=12)$ and DCS+SCOP $(n=10)$.

\section{Behavioral testing}

As depicted in Figure 2, statistically significant between-group differences in the percentage of trained food eaten were found $\left[\mathrm{F}_{[3,41]}=8.858, \mathrm{P}=0.0001\right]$. Contrast analyses showed statistically significant differences between SCOP and the remaining groups: VEH ( $\mathrm{P}=0.006), \mathrm{DCS}(\mathrm{P}=0.002)$ and DCS+SCOP $(\mathrm{P}<0.0001)$. SCOP rats apparently exhibited no preference for the trained food since it was not significantly different from chance level $\left(\mathrm{t}_{[11]}=-1.675, \mathrm{P}=0.122\right)$ whereas the other groups performed significantly above chance level ( $\mathrm{t}_{[9]}$ 's $>3.41$ and P's $\left.<0.01\right)$. The analysis of the social interaction measures showed no statistically significant group effects in any of the variables (muzzle: $\mathrm{F}_{[3,41]}=1.880, \mathrm{P}=0.149$; body: $\mathrm{F}_{[3,41]}=1.522, \mathrm{P}=0.224$; anogenital: $\mathrm{F}_{[3,41]}=0.785, \mathrm{P}=0.510$; fighting: $\mathrm{F}_{[3,41]}=0.724, \mathrm{P}=0.544$ and grooming $\left.\mathrm{F}_{[3,41]}=0.169, \mathrm{P}=0.917\right)$. Nor were there any statistically significant correlations between such variables and the percentage of trained food.

No statistically significant between-group differences were observed in exploration of both food jars (jar climbs) $\left(\mathrm{F}_{[3,41]}=0.472, \mathrm{P}=0.706\right)$ and in the total amount of food consumed during the test session $\left(\mathrm{F}_{[3,41]}=0.257, \mathrm{P}=0.856\right)$. In the analysis of possible neophobic effects, mixed ANOVA showed a significant effect of food $\left(\mathrm{F}_{[1,38]}=4.611, \mathrm{P}=0.038\right)$ but no significant effects of group $\left(\mathrm{F}_{[3,38]}=1.081\right.$, $\mathrm{P}=0.369)$ or group $\mathrm{x}$ food interaction $\left(\mathrm{F}_{[3,38]}=1.614, \mathrm{P}=0.202\right)$.

The performance in this task did not seem to be related to changes in olfactory sensitivity as no statistically significant between-group differences were observed when the latency to find a buried sweet-smelling cookie was analyzed $24 \mathrm{~h}$ after injections $\left(\mathrm{F}_{[3,41]}=0.139, \mathrm{P}=0.936\right)$.

\section{Experiment 2}

After a 20-min basal period and stable fEPSP responses, the different drugs [DCS 50 $\mu \mathrm{M}$

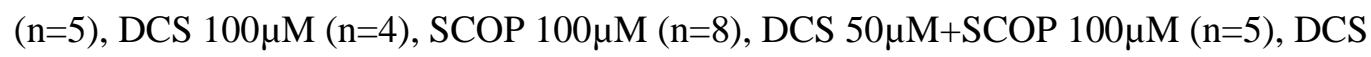
$100 \mu \mathrm{M}+\mathrm{SCOP} 100 \mu \mathrm{M}(\mathrm{n}=4)]$ were added into the KRB in the corresponding groups. The application of DCS to hippocampal slices induced a slight potentiation of basal synaptic transmission in all the groups (see Figures 3 and 4). The ANOVA comparing the fEPSP responses $20 \mathrm{~min}$ after drug 
perfusion (time before HFS) versus the 20-min of baseline period of the VEH group showed statistically significant differences in the following groups: DCS $50 \mu \mathrm{M}\left(\mathrm{F}_{[1,11]}=8.862, \mathrm{P}=0.014\right), \mathrm{DCS}$ $100 \mu \mathrm{M}\left(\mathrm{F}_{[1,10]}=13.824, \mathrm{P}=0.005\right), \mathrm{DCS} 50 \mu \mathrm{M}+\mathrm{SCOP} 100 \mu \mathrm{M}\left(\mathrm{F}_{[1,13]}=6.514, \mathrm{P}=0.025\right)$ and $\mathrm{DCS}$ $100 \mu \mathrm{M}+\mathrm{SCOP} 100 \mu \mathrm{M}\left(\mathrm{F}_{[1,12]}=5.258, \mathrm{P}=0.043\right)$, but not in the SCOP $100 \mu \mathrm{M}$ group $\left(\mathrm{F}_{[1,14]}=0.467\right.$, $\mathrm{P}=0.506)($ Figure 5A).

After HFS and following 20 min of drug perfusion, the effects of the drugs on LTP were analyzed (Figures 3 and 4). The ANOVA of the last 10 minutes of recording showed that the group $\left(\mathrm{F}_{[5,31]}=3.919, \mathrm{P}=0.007\right)$ and time $\left(\mathrm{F}_{[9,279]}=6.434, \mathrm{P}<0.0001\right)$ factors were statistically significant, but not the interaction group $\mathrm{x}$ time $\left[\mathrm{F}_{[45,279]}=1.104, \mathrm{P}=0.311\right]$. Specifically, the group differences were found between the SCOP $100 \mu \mathrm{M}$ group and the remaining groups: VEH (P=0.024), DCS 50 $\mu \mathrm{M}$ $(\mathrm{P}<0.0001)$, DCS $100 \mu \mathrm{M}(\mathrm{P}=0.003)$, DCS 50 $\mu \mathrm{M}+\mathrm{SCOP} 100 \mu \mathrm{M}(\mathrm{P}=0.05)$ and DCS $100 \mu \mathrm{M}+\mathrm{SCOP}$ $100 \mu \mathrm{M}(\mathrm{P}=0.033)$ (Figures 3 and 4). Hence, the administration of DCS offset the deficit on the LTP maintenance induced by SCOP. Additionally, the analysis of the potentiation percentage in the final recording indicated statistically significant between-group differences $\left[\mathrm{F}_{[5,31]}=2.866, \mathrm{P}=0.0306\right]$. The differences were appreciated between the SCOP 100 $\mu \mathrm{M}$ group vs. VEH $(\mathrm{P}=0.019)$, DCS $50 \mu \mathrm{M}+\mathrm{SCOP} 100 \mu \mathrm{M}(\mathrm{P}=0.05)$, DCS $100 \mu \mathrm{M}+\mathrm{SCOP} 100 \mu \mathrm{M}(\mathrm{P}=0.05)$, DCS 50 $\mu \mathrm{M}(\mathrm{P}=0.002)$ and DCS $100 \mu \mathrm{M}(\mathrm{P}=0.023)$ (Figure 5B).

\section{DISCUSSION}

The present study provides behavioral and electrophysiological evidence that the potentiation of glutamatergic activity in the hippocampus, with microinfusion of the partial NMDAR agonist DCS, rescues relational memory deficits and in-vitro LTP maintenance impairment, both induced by the administration of the muscarinic antagonist SCOP. In the experiment 1, vHPC administration of DCS prior to social interaction prevented the SCOP-induced amnesia in the 24-h memory test, as the trained food preference of the DCS+SCOP group was significantly higher than the chance level and that of the SCOP group, but not thanVEH and DCS groups. These results cannot be attributed to alterations in olfactory perception, social investigation, neophobic responses or motor activity. The counteraction of SCOP-induced deficits is consistent with early reports suggesting that the hippocampus may be a 
critical region for the action of DCS in the reversion of SCOP-induced impairments in spatial working memory (Kishi et al., 1998; Ohno and Watanabe, 1996). Previous research also showed that the systemic pre-learning injection of DCS attenuated SCOP-induced deficits both in spatial (Pitkänen et al., 1995) and non-spatial paradigms (Andersen et al., 2002). Therefore, the present results provide more evidence as to a potential pervasive role of DCS in the reversion of memory deficits induced by different causes, such as cholinergic or glutamatergic dysfunction (Kawabe et al., 1998), aging (Aura and Riekkinen, 2000), stress (Yamamoto et al., 2008), traumatic brain injury and hippocampal, medial septal or thalamic lesions (Villarejo-Rodríguez et al., 2013; Yaka et al., 2007). Data from experiment 2 showed that the two doses of DCS enhanced the potentiation percentage in SCOP-treated slices at the end of registering, which would suggest that the cellular mechanism underlying the reversion of SCOP-induced amnesia in STFP may involve long-term synaptic plasticity. Our results show that both basal and post HFS synaptic transmission under 50 or $100 \mu \mathrm{M}$ of DCS reached a ceiling effect in control slices. Moreover, it could be considered that $100 \mu \mathrm{M}$ of DCS in SCOP-treated slices also came to a ceiling because HFS produced an identical potentiation in all the experimental situations due to the saturated-LTP induced. Nevertheless, the maintenance of the potentiation is the really interesting effect of DCS in the reversion of the non-lasting LTP under SCOP treatment. The present physiological data agree with studies showing that DCS can reverse deficits in hippocampal LTP induced by head injury (Yaka et al., 2007), NCAM-deficiency (Kochlamazashvili et al., 2012), or aging (Billard and Rouaud, 2007). It has been shown that DCS not only facilitated NMDAR activation and synaptic plasticity in young rats, but was significantly more potent in aged animals, thus reversing the age-related deficit of NMDAR-mediated LTP and long-term depression (LTD) (Billard and Rouaud, 2007).

As to the behavioral effects of DCS in non-SCOP treated rats, the present study showed that DCS did not improve STFP memory, which is consistent with data from previous experiments showing that DCS in the PLC ameliorated SCOP-induced deficits in the STFP task, but did not enhance memory when injected in control rats (Portero-Tresserra et al., 2013a). Such findings would suggest, as discussed previously (Portero-Tresserra et al., 2013a), that DCS may enhance implicit or procedural tasks, but that its facilitative influence on relational paradigms seems to be more restricted. 
Another factor that may be considered to explain the effects of DCS in control subjects is the targeted cognitive process, since some studies showed a beneficial effect of DCS specifically in the extinction of fear conditioning (Ren et al., 2013). In contrast, in the present experiment DCS administration probably affected the original learning/early consolidation as the drug was injected prior to STFP training. Regarding the physiological effects of DCS, data from experiment 2 showed that DCS in non SCOP-treated slices did not enhance hippocampal LTP. This contrasts with an earlier study showing that DCS facilitated synaptic plasticity of glutamatergic transmission in the CA1 field, lowering the threshold to induce a lasting potentiation (Rouaud and Billard, 2003). Methodological factors may explain divergence in findings since the Rouaud and Billard (2003) study used a protocol inducing a shorter potentiation. The present data also indicated that the administration of DCS in CA1, before HFS, increased the magnitude of the fEPSP recorded both in slices treated and untreated with SCOP. Thus, all the DCS groups showed an increased percentage in the fEPSP slope from the 20-min baseline to the initial 20-min drug presence condition $(7.78 \%, 11.85 \%, 6.38 \%$ and $7.78 \%)$. These findings may be interpreted as an enhancement in synaptic transmission (although it did not significantly endure after HFS), which would agree with the Rouaud and Billard (2003) work indicating that DCS facilitated NMDAR-mediated fEPSPs in the CA1 region of rat hippocampal slices. Such results found in the CA1 hippocampal region also confirm an early report demonstrating that DCS increased in vivo the excitability of dentate granule cells (Pitkänen et al., 1994). Nevertheless, the fact that DCS enhanced NMDAR-dependent synaptic potentials but not LTP or behavioral memory in non-SCOP groups was not wholly unexpected as earlier studies indicated that DCS had a more robust effect in animals showing a certain cognitive deficit (Aura and Riekkinen, 2000; Billard and Rouaud, 2007).

The present results also confirm that the infusion of SCOP $(40 \mu \mathrm{g} / \mathrm{hemisphere})$ in the vHPC immediately after the STFP acquisition produced a striking deficit in the $24 \mathrm{~h}$-memory test, detected by the poor performance of the SCOP group, which did not differ from the chance level and was significantly worse than that of the VEH group. Such data corroborate the contribution of muscarinic receptors in the consolidation of this social form of relational memory as STFP impairments had also been observed when a lower dose of SCOP $(20 \mu \mathrm{g})$ was administered in the vHPC or the PLC 
(Carballo-Marquez et al., 2009; Portero-Tresserra et al., 2013a). Interestingly, muscarinic receptors appear to contribute to the recognition of socially relevant odors but not neutral odors, since systemic administration of SCOP significantly disrupted urine estrous odor but no mint odor habituation (Miranda et al. 2009). The importance for STFP acquisition of the cholinergic transmission has also been demonstrated in a microdialysis study showing a significant increase $(200 \%)$ of ACh release in the vHPC after exposure to a rat that had eaten the novel food, which was not found in the PLC (Gold et al., 2011). Such effects are consistent with the involvement of the cholinergic system in regulating the salience of odors during active sensing of the olfactory environment (Rothermel et al., 2014), which agrees with the proposed role of ACh in attentional mechanisms. The current electrophysiological data also demonstrated that the SCOP $(100 \mu \mathrm{M})$ perfusion in hippocampal slices significantly suppressed the LTP maintenance in CA1 synapses, as indicated by the lower percentage of potentiation in the last $10 \mathrm{~min}$ of recording by the SCOP group vs. the remaining groups, thus confirming previous findings (Sánchez et al., 2009). Moreover, detrimental effects of SCOP on LTP have also been found in CA1 synapses in vivo (Ovsepian et al., 2004) and in corticostriatal synapses (Calabresi et al., 1999). In agreement with the above, it has been reported that muscarinic receptor activation facilitates the induction of LTP in rat hippocampal slices (Buchanan et al., 2010), which would corroborate that such receptors are a crucial event in this form of synaptic plasticity.

Taken together, the results from both experiments point to an interactive relationship between glutamatergic and cholinergic transmission in memory modulation, previously suggested by behavioral pharmacological studies (Ohno and Watanabe, 1996). For instance, concomitant administration of sub-threshold doses of SCOP and NMDA antagonists induced amnesia in inhibitory avoidance and spatial learning (Khakpai et al., 2012) and sub-effective doses of NMDA and physostigmine exerted memory-enhancing properties (Jafari-Sabet, 2006). There is also evidence at the cellular level indicating the interaction between cholinergic and glutamatergic systems in mediating memory processes on the grounds of their involvement in synaptic plasticity. Markram and Segal (1990) demonstrated that the administration of ACh increases the probability of generating hippocampal NMDA-dependent LTP. Furthermore, the activation of muscarinic receptors can also lead to LTD of NMDAR-mediated synaptic transmission (Jo et al., 2010). In this sense, it has been 
shown that M1 muscarinic receptors induce the opening of NMDA receptors through the inhibition of SK channels (Buchanan et al., 2010). Moreover, cooperation between cholinergic and glutamatergic receptors would seem to be essential to induce BDNF-dependent long-lasting memory storage (Navakkode and Korte, 2012). Additionally, other mechanisms by which SCOP may disrupt LTP have been suggested, such as down-regulation of protein kinase C and nitric oxide (Saraf et al., 2010), which are required for LTP induction and maintenance (Knafo et al., 2012). Therefore, it is widely known that muscarinic ACh receptors and NMDAR are important for learning and memory, although the specific manner in which they interact is less understood (Doguc et al., 2012). Indeed, few studies had assessed the brain regions where such an interaction may take place (Khakpai et al., 2012) or the function of intracerebral DCS as a preventive treatment for mnemonic deficits produced by cholinergic deficiency (Portero-Tresserra et al., 2013a), which enhances the significance of the current research.

In summary, in the present study we found a correspondence between behavioral and cellular findings. In both experiments, the administration of DCS was able to rescue SCOP-induced impairments since the DCS+SCOP groups showed comparable results in STFP memory and electrophysiological recording. Consequently, DCS effects on STFP memory may be interpreted in terms of enhancing hippocampal LTP, which may be considered as a crucial element in memory or other forms of experience-dependent plasticity. Although there is no general consensus regarding LTP as a model for the synaptic changes that contribute to the neural circuit modifications underlying all types of experience-dependent plasticity, accumulating evidence would suggest that this mechanism has important functional roles in many brain areas and in different circumstances (Malenka, 2003). Our results, therefore, provide functional evidence that DCS may be helpful in reducing memory impairments associated with cholinergic depletion, probably by enhancing LTP. Hence, our findings support the use of NMDA-glycine site agonists, such as DCS, as potential treatments for the alleviation of memory alteration occurring in neurodegenerative or neurological diseases.

\section{AUTHOR DISCLOSURE}


Funding for this study was provided by Ministerio de Ciencia e Innovación (PSI2011-26862; FPI grant to MP-T: BES-2009-015395; SAF2011-25300) which had no further role in study design, collection, analysis and interpretation of data, writing of the report, and decision to submit the paper for publication.

Portero-Tresserra, Vale-Martínez designed the first experiment, undertook the statistical analyses, and managed the literature. Portero-Tresserra and Del Olmo designed and carried out the second experiment. Portero-Tresserra, Vale-Martínez, Martí-Nicolovius and Guillazo-Blanch wrote the first draft of the manuscript. All authors contributed to and have approved the final manuscript.

There are no conflicts of interest.

\section{ACKOWLEDGEMENTS}

The authors wish to thank Dr. Emilio Ambrosio for sharing the electrophysiology equipment and Gerald-Patrick Fannon for his support with the English-language editing. 


\section{REFERENCES}

Andersen, J.M., Lindberg, V., Myhrer, T., 2002. Effects of scopolamine and D-cycloserine on non-spatial reference memory in rats. Behav Brain Res. 129, 211-216.

Aura, J., Riekkinen, P., 2000. Pre-training blocks the improving effect of tetrahydroaminoacridine and Dcycloserine on spatial navigation performance in aged rats. Eur J Pharmacol. 390, 313-8.

Billard, J.M., Rouaud, E., 2007. Deficit of NMDA receptor activation in CA1 hippocampal area of aged rats is rescued by D-cycloserine. Eur J Neurosci. 25, 2260-8.

Buchanan, K.A., Petrovic, M.M., Chamberlain, S.E., Marrion, N.V., Mellor, J.R., 2010. Facilitation of long-term potentiation by muscarinic M(1) receptors is mediated by inhibition of SK channels. Neuron. 68, 948-63.

Calabresi, P., Centonze, D., Gubellini, P., Bernardi, G., 1999. Activation of M1-like muscarinic receptors is required for the induction of corticostriatal LTP. Neuropharmacology. 38, 323-6.

Carballo-Marquez, A., Vale-Martinez, A., Guillazo-Blanch, G., Marti-Nicolovius, M., 2009. Muscarinic receptor blockade in ventral hippocampus and prelimbic cortex impairs memory for socially transmitted food preference. Hippocampus. 19, 446-55.

Carballo-Marquez, A., Vale-Martinez, A., Guillazo-Blanch, G., Torras-Garcia, M., Boix-Trelis, N., Marti-Nicolovius, M., 2007. Differential effects of muscarinic receptor blockade in prelimbic cortex on acquisition and memory formation of an odor-reward task. Learn Mem. 14, 616-624.

Countryman, R.A., Orlowski, J.D., Brightwell, J.J., Oskowitz, A.Z., Colombo, P.J., 2005. CREB phosphorylation and c-Fos expression in the hippocampus of rats during acquisition and recall of a socially transmitted food preference. Hippocampus. 15, 56-67.

Doguc, D.K., Delibas, N., Vural, H., Altuntas, I., Sutcu, R., Sonmez, Y., 2012. Effects of chronic scopolamine administration on spatial working memory and hippocampal receptors related to learning. Behav Pharmacol. 23(8), 762-70.

Eichenbaum, H., 2000. A cortical-hippocampal system for declarative memory. Nat Rev Neurosci. 1, 4150.

Falsafi, S.K., Deli, A., Höger, H., Pollak, A., Lubec, G., 2012. Scopolamine administration modulates muscarinic, nicotinic and NMDA receptor systems. PloS one. 7, e32082. 
Gold, P.E., Countryman, R.A., Dukala, D., Chang, Q., 2011. Acetylcholine release in the hippocampus and prelimbic cortex during acquisition of a socially transmitted food preference. Neurobiol Learn Mem. 96, 498-503.

Jafari-Sabet, M., 2006. NMDA receptor blockers prevents the facilitatory effects of post-training intradorsal hippocampal NMDA and physostigmine on memory retention of passive avoidance learning in rats. Behav Brain Res. 169, 120-7.

Jo, J., Son, G.H., Winters, B.L., Kim, M.J., Whitcomb, D.J., Dickinson, B.A., Lee, Y.B., Futai, K., Amici, M., Sheng, M., et al. 2010. Muscarinic receptors induce LTD of NMDAR EPSCs via a mechanism involving hippocalcin, AP2 and PSD-95. Nat Neurosci. 13, 1216-24.

Kalisch, R., Holt, B., Petrovic, P., De Martino, B., Klöppel, S., Büchel, C., Dolan, R.J., 2009. The NMDA agonist D-cycloserine facilitates fear memory consolidation in humans. Cereb Cortex. 19, 187-96.

Kawabe, K., Yoshihara, T., Ichitani, Y., Iwasaki, T., 1998. Intrahippocampal D-cycloserine improves MK-801-induced memory deficits: radial-arm maze performance in rats. Brain Res. 814, 226-230.

Khakpai, F., Nasehi, M., Haeri-Rohani, A., Eidi, A., Zarrindast, M.R., 2012. Scopolamine induced memory impairment; possible involvement of NMDA receptor mechanisms of dorsal hippocampus and/or septum. Behav Brain Res. 231, 1-10.

Kishi, A., Ohno, M., Watanabe, S., 1998. Concurrent activation of hippocampal glycine and polyamine sites of the N-methyl-D-aspartate receptor synergistically reverses working memory deficits in rats. Neurosci Lett. 257, 131-4.

Klinkenberg, I., Blokland, A., 2010. The validity of scopolamine as a pharmacological model for cognitive impairment: a review of animal behavioral studies. Neurosci Biobehav Rev. 34, 130750.

Knafo, S., Venero, C., Sánchez-Puelles, C., Pereda-Peréz, I., Franco, A., Sandi, C., Suárez, L.M., Solís, J.M., Alonso-Nanclares, L., Martín, E.D., et al. 2012. Facilitation of AMPA receptor synaptic delivery as a molecular mechanism for cognitive enhancement. PLoS biology. 10, e1001262.

Kochlamazashvili, G., Bukalo, O., Senkov, O., Salmen, B., Gerardy-Schahn, R., Engel, A.K., Schachner, M., Dityatev, A., 2012. Restoration of Synaptic Plasticity and Learning in Young and Aged NCAM-Deficient Mice by Enhancing Neurotransmission Mediated by GluN2A-Containing NMDA Receptors. J Neurosci. 32, 2263-2275. 
Lee, Y.S., Silva, A.J., 2009. The molecular and cellular biology of enhanced cognition. Brain Res. 10, $126-140$.

Malenka, R.C., 2003. The long-term potential of LTP. Nat Rev Neurosci. 4, 923-6.

Markram, H., Segal, M., 1990. Long-lasting facilitation of excitatory postsynaptic potentials in the rat hippocampus by acetylcholine. J Physiol. 427, 381-93.

Miguéns, M., Coria, S.M., Higuera-Matas, A., Fole, A., Ambrosio, E., Del Olmo, N., 2011.

Depotentiation of hippocampal long-term potentiation depends on genetic background and is modulated by cocaine self-administration. Neuroscience. 187, 36-42.

Miranda, M.I., Ortiz-Godina, F., García, D., 2009. Differential involvement of cholinergic and betaadrenergic systems during acquisition, consolidation, and retrieval of long-term memory of social and neutral odors. Behav Brain Res. 202, 19-25.

Navakkode, S., Korte, M., 2012. Cooperation between cholinergic and glutamatergic receptors are essential to induce BDNF-dependent long-lasting memory storage. Hippocampus. 22, 335-46.

Norberg, M.M., Krystal, J.H., Tolin, D.F., 2008. A meta-analysis of D-cycloserine and the facilitation of fear extinction and exposure therapy. Biol Psychiatry. 63, 1118-26.

Ohno, M., Watanabe, S., 1996. D-cycloserine, a glycine site agonist, reverses working memory failure by hippocampal muscarinic receptor blockade in rats. Eur J Pharmacol. 318, 267-271.

Onur, O.A., Schlaepfer, T.E., Kukolja, J., Bauer, A., Jeung, H., Patin, A., Otte, D.M., Shah, N.J., Maier, W., Kendrick, K.M., et al. 2010. The N-methyl-D-aspartate receptor co-agonist D-cycloserine facilitates declarative learning and hippocampal activity in humans. Biol Psychiatry. 67, 1205-11.

Ovsepian, S.V., Anwyl, R., Rowan, M.J., 2004. Endogenous acetylcholine lowers the threshold for longterm potentiation induction in the CA1 area through muscarinic receptor activation: in vivo study. Eur J Neurosci. 20, 1267-75.

Paxinos, G., Watson, C., 1997. The rat brain in stereotaxic coordinates. San Diego: Academic Press.

Peters, J., De Vries, T.J., 2013. D-cycloserine administered directly to infralimbic medial prefrontal cortex enhances extinction memory in sucrose-seeking animals. Neuroscience. 230, 24-30.

Pitkänen M, Sirviö J, Lahtinen H, Koivisto E, Riekkinen P. 1994. d-Cycloserine, a partial agonist at the glycine site, enhances the excitability of dentate granule cells in vivo in rats. Eur J Pharmacol. 253(1-2), 125-9. 
Pitkänen, M., Sirviö, J., MacDonald, E., Niemi, S., Ekonsalo, T., Riekkinen, P., 1995. The effects of Dcycloserine and MK-801 on the performance of rats in two spatial learning and memory tasks. Eur Neuropsychopharmacol. 5, 457-63.

Portero-Tresserra, M., Cristóbal-Narváez, P., Martí-Nicolovius, M., Guillazo-Blanch, G., Vale-Martínez, A., 2013a. D-cycloserine in prelimbic cortex reverses scopolamine-induced deficits in olfactory memory in rats. Plos One. 8(8),e70584.

Portero-Tresserra, M., Martí-Nicolovius, M., Guillazo-Blanch, G., Boadas-Vaello, P., Vale-Martínez, A., 2013b. D-Cycloserine in the Basolateral Amygdala Prevents Extinction and Enhances

Reconsolidation of Odor-Reward Associative Learning in Rats. Neurobiol Learn Mem. 100, 1-11.

Ren, J., Li, X., Zhang, X., Li, M., Wang, Y., Ma, Y., 2013. The effects of intra-hippocampal microinfusion of D-cycloserine on fear extinction, and the expression of NMDA receptor subunit NR2B and neurogenesis in the hippocampus in rats. Prog Neuropsychopharmacol Biol Psychiatry. $44,257-64$.

Rothermel, M., Carey, R.M., Puche, A., Shipley, M.T., Wachowiak, M., 2014. Cholinergic inputs from basal forebrain add an excitatory bias to odor coding in the olfactory bulb. J Neurosci. 34, 465464.

Ross, R.S., Eichenbaum, H., 2006. Dynamics of hippocampal and cortical activation during consolidation of a nonspatial memory. J Neurosci. 26, 4852-4859.

Rouaud, E., Billard, J.M., 2003. D-cycloserine facilitates synaptic plasticity but impairs glutamatergic neurotransmission in rat hippocampal slices. Br J Pharmacol. 140, 1051-6.

Sánchez, G., Alvares, L.D.O., Oberholzer, M.V., Genro, B., Quillfeldt, J., da Costa, J.C., Cerveñansky, C., Jerusalinsky, D., Kornisiuk, E., 2009. M4 muscarinic receptors are involved in modulation of neurotransmission at synapses of Schaffer collaterals on CA1 hippocampal neurons in rats. J Neurosci Res. 87, 691-700.

Saraf, M.K., Anand, A., Prabhakar, S., 2010. Scopolamine induced amnesia is reversed by Bacopa monniera through participation of kinase-CREB pathway. Neurochem Res. 35, 279-87.

Tsai, G.E., Falk, W.E., Gunther, J., Coyle, J.T., 1999. Improved cognition in Alzheimer's disease with short-term D-cycloserine treatment. Am J Psychiatry. 156, 467-469. 
Vale-Martinez, A., Baxter, M.G., Eichenbaum, H., 2002. Selective lesions of basal forebrain cholinergic neurons produce anterograde and retrograde deficits in a social transmission of food preference task in rats. Eur J Neurosci. 16, 983-998.

Villarejo-Rodríguez, I., Boadas-Vaello, P., Portero-Tresserra, M., Vale-Martínez, A., Martí-Nicolovius, M., Guillazo-Blanch, G., 2013. Learning deficits in an odor reward-task induced by parafascicular thalamic lesions are ameliorated by pretraining d-cycloserine in the prelimbic cortex. Behav Brain Res. 238, 289-92.

Villarejo-Rodríguez, I., Vale-Martínez, A., Guillazo-Blanch, G., Martí-Nicolovius, M., VillarejoRodriguez, I., Vale-Martinez, A., Marti-Nicolovius, M., 2010. D-cycloserine in prelimbic cortex enhances relearning of an odor-reward associative task. Behav Brain Res. 213, 113-6.

Walker, D.L., Ressler, K.J., Lu, K.T., Davis, M., 2002. Facilitation of conditioned fear extinction by systemic administration or intra-amygdala infusions of D-cycloserine as assessed with fearpotentiated startle in rats. J Neurosci. 22, 2343-51.

Yaka, R., Biegon, A., Grigoriadis, N., Simeonidou, C., Grigoriadis, S., Alexandrovich, A.G., Matzner, H., Schumann, J., Trembovler, V., Tsenter, J., et al. 2007. D-cycloserine improves functional recovery and reinstates long-term potentiation (LTP) in a mouse model of closed head injury. FASEB J. 21, 2033-41.

Yamamoto, S., Morinobu, S., Fuchikami, M., Kurata, A., Kozuru, T., Yamawaki, S., 2008. Effects of single prolonged stress and D-cycloserine on contextual fear extinction and hippocampal NMDA receptor expression in a rat model of PTSD. Eur Neuropsychopharmacol. 33, 2108-2116. 


\section{FIGURE LEGENDS}

FIGURE 1. Histology. (A) Photomicrograph (10x) of acetylcholinesterase staining at the vHPC area (AP, $5.20 \mathrm{~mm}$ posterior to Bregma) showing the cannulae tracks and the microinjector tips of a representative subject. (B) Micro-injector tip placements throughout the rostral-caudal extent vHPC for each group: VEH (open circles), DCS (filled circles), SCOP (open triangles), and DCS+SCOP (filled triangles). [CA2: field CA2 of hippocampus, CA3: field CA3 of hippocampus, DG: dentate gyrus]

FIGURE 2. Effects of D-cycloserine on STFP. Percentage of the trained food selected, expressed as the mean percentage $( \pm$ SEM) of the total amount of food consumed in the STFP 24h-test $(* * \mathrm{P}<0.01, * * * \mathrm{P}<0.001)$.

FIGURE 3. Effects of D-cycloserine on LTP. The symbols represent the fEPSP slope values

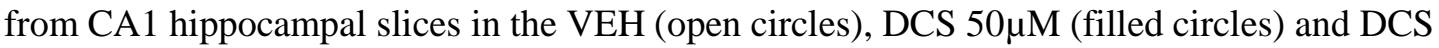
$100 \mu \mathrm{M}$ (filled triangles) groups. The upper traces are the averages of the fEPSPs recorded during the basal period (a, thin trace), following high frequency stimulation (HFS, indicated by arrows) (b, thick trace) and final record (c, dash trace). Calibration: $1 \mathrm{mV}, 2 \mathrm{~ms}$.

FIGURE 4. Effects of D-cycloserine and scopolamine on LTP. The symbols represent the fEPSP slope values from CA1 hippocampal slices in the DCS $100 \mu \mathrm{M}+\mathrm{SCOP} 100 \mu \mathrm{M}$ (filled

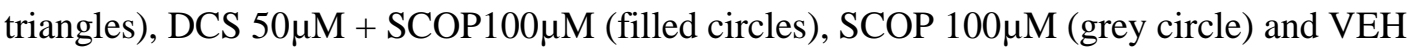
(open circles) groups. The upper traces are the averages of the fEPSPs recorded during the basal period (a, thin trace), after HFS (b, thick trace) and final record (c, dash trace). Calibration: 1 $\mathrm{mV}, 2 \mathrm{~ms}$.

FIGURE 5. Effects of D-cycloserine before and after high-frequency stimulation. (A) Mean fEPSP slope during the 20-min basal period for VEH group and the 20-min drug perfusion prior 
HFS for the remaining groups $(* \mathrm{P}<0.05$ and $* * \mathrm{P}<0.01)$. (B) Mean fEPSP slope (\% Control) during the last 10 min of recording in control medium $(* \mathrm{P}<0.05$ and $* * \mathrm{P}<0.01)$. 